BEREH
AUG $0>1998$
OSTI GA-A22880
CONF- $980560-$

CARBON INFLUX IN HE AND D PLASMAS IN DIII-D

\author{
by \\ W.P. WEST, N.H. BROOKS, M.E. FENSTERMACHER, R.C. ISLER, \\ G.L. JACKSON, C.J. LASNIER, A. RAMSEY, M.R. WADE, \\ D.G. WHYTE and R.D. WOOD
}

distaisution of this document is unLMTted ph MASTER

JULY 1998 


\section{DISCLAIMER}

This report was prepared as an account of work sponsored by an agency of the United States Government. Neither the United States Government nor any agency thereof, nor any of their employees, makes any warranty, express or implied, or assumes any legal liability or responsibility for the accuracy, completeness, or usefulness of any information, apparatus, product, or process disclosed, or represents that its use would not infringe privately owned rights. Reference herein to any specific commercial product, process, or service by trade name, trademark, manufacturer, or otherwise, does not necessarily constitute or imply its endorsement, recommendation, or favoring by the United States Government or any agency thereof. The views and opinions of authors expressed herein do not necessarily state or reflect those of the United States Government or any agency thereof. 


\section{DISCLAIMER}

Portions of this document may be illegible electronic image products. Images are produced from the best available original document. 
GA-A22880

\title{
CARBON INFLUX IN HE AND D PLASMAS IN DIII-D
}

\author{
by
}

W.P. WEST, N.H. BROOKS, M.E. FENSTERMACHER, † R.C. ISLER, $\ddagger$ G.L. JACKSON, C.J. LASNIER, † A. RAMSEY,, M.R. WADE, $\ddagger$ D.G. WHYTE, $\triangle$ and R.D. WOOD ${ }^{\prime}$

This is a preprint of a paper to be presented at the 13th International Conference on Plasma Surface Interactions in Controlled Fusion Devices, May 18-23, 1998, San Diego, California and to be published in Journal of Nuclear Materials.

\author{
†Lawrence Livermore National Laboratory \\ ¥Oak Ridge National Laboratory \\ $\vartheta$ Princeton Plasma Physics Laboratory \\ $\Delta$ University of California, San Diego
}

Work supported by

the U.S. Department of Energy

under Contracts DE-AC03-89ER51114, DE-AC05-960R22464, W-7405-ENG-48, DE-AC02-76CH03073, and Grant DE-FG03-95ER54294

\author{
GA PROJECT 3466 \\ JULY 1998
}




\begin{abstract}
Differences in the carbon behavior between He and D plasmas during VH-mode, L-mode and L-mode with excess gas puffing are reported and inferences on the importance of the various carbon sources during these modes of operation are discussed. During a VH-mode phase, VUV and visible charge exchange spectroscopy indicates that for both $\mathrm{He}$ and $\mathrm{D}$ operation the carbon behavior is very similar. In the edge plasma, carbon build up is quite rapid, and the carbon influx represents a large fraction of the total plasma density increase until the termination of the $\mathrm{VH}$ phase. During cold divertor operation induced by puffing the primary fueling gas, D and He discharges show a difference in the carbon behavior. The core carbon density is seen to be approximately constant during a D discharge as it transitions from an attached to a cold divertor. However in a $\mathrm{He}$ discharge, the core carbon density disappears soon after the cold divertor transition. Arguments are made that the primary carbon source in the ELM free $\mathrm{H}$-mode period is physical sputtering by ion impact at the divertor strike point. In L-mode, both attached and cold divertor, the primary source is from the divertor region and two possibilities for this source are chemical sputtering or charge exchange neutral sputtering. Existing data supports charge exchange neutrals as dominant.
\end{abstract}


$\gamma$ 


\section{INTRODUCTION}

Carbon is almost always the dominant impurity in DIII-D, and can have important effects on the particle and power balance of the plasma. DIII-D has an all carbon wall $[1,2]$, so there are several possible sources for carbon influx, including: 1) physical sputtering from divertor strike points, 2) chemical sputtering from divertor walls, and 3) physical and chemical sputtering from main chamber and divertor walls by energetic charge exchange neutrals. A reduction in the impurity content of DIII-D plasmas might be achieved through changes to the plasma facing materials, however an improved understanding of the importance of the various sources to core plasma carbon influx is needed to choose materials wisely.

Plasmas in DIII-D vary widely in edge properties, and these variations are likely to influence which of the possible sources are important. For example, high performance plasmas on DIII-D are typically transient, with low edge density, high edge temperatures and conduction limited transport along the open field lines to the divertor strike plate. Contrastingly, radiative divertor experiments are typically carried out in plasmas with high edge denisty, in a high recycling or detached divertor mode. ELM free $\mathrm{H}$-mode exhibits the key carbon influx problem characteristic of high performance experiments on DIII-D. Carbon build up in the core plasma is dramatic during ELM free $\mathrm{H}$ and $\mathrm{VH}$ confinement phases. $Z_{\text {eff }}$ approaching six is sometimes observed at $\rho$ $>0.7$ before the termination of a VH-mode phase. It has been determined that core carbon influx accounts for a substantial fraction of the density increase during VH and ELM free H-modes [2]. In radiative divertor experiments, heavy $\mathrm{D}_{2}$ puffing is used on DIII-D to produce a discharge with high divertor density, low divertor temperatures, and a high radiation fraction [3]. Spectroscopic analysis of these highly radiating discharges shows that carbon is the dominant radiator [1]. Analysis of DIII-D wall tiles and of graphite test samples has shown that deuterium plasma operation on graphite tiles produces significant changes in the first wall surface conditions, and that carbon/deuterium redeposition appears to be a major part of wall deconditioning $[4,5]$. Understanding carbon sources and transport could lead to intellegent changes in the DIII-D first wall and improvements in the performance of DIII-D plasmas.

The all graphite wall provides multiple carbon sources, but the source strength alone does not determine its contribution to core plasma contamination. To contaminate the core plasma, carbon must be transported from its' origin at the wall through the SOL, past the seperatrix and into the core plasma. Carbon ions from divertor sources must transport along field lines to the midplane and subsequently into the core. Neutral carbon from the divertor or from the main chamber walls must pass through the SOL plasma to contaminate the core plasma directly. Thus, the relative 
importance of the various sources as well as the transport of neutrals and ions are dependent on many details of the edge and divertor plasma conditions.

Helium plasmas can be produced in DIII-D by changing the primary fueling gas from the usual deuterium to helium along with careful wall conditioning. Chemical sputtering in these helium plasmas should be greatly reduced. Charge exchange processes at the plasma edge, leading to an outflux of energetic charge exchange neutrals that will sputter carbon from the walls, should also change somewhat when the primary ion, and therefore the recycling gas, is changed. 


\section{DIII-D DIAGNOSTICS FOR CARBON REFLUX STUDIES}

Diagnostic systems which are very important to this study are briefly discussed in this section. Recycling emission from both $\mathrm{D}\left(\mathrm{D}_{\alpha}\right.$ at $\left.656 \mathrm{~nm}\right)$ and $\mathrm{He}(\mathrm{HeII}$ at $468 \mathrm{~nm}$ ) is measured simultaneously along several identical chords in both the upper and lower divertor. Emission spectra from low charge states of impurities in the divertor are measured in both the visible and VUV. The divertor VUV SPRED survey spectrometer views the lower divertor vertically from above with a spot width of about $2 \mathrm{~cm}$ at the divertor floor [1]. The visible Multichord Divertor Spectrometer (MDS) has seven chords viewing the lower divertor from above, each with a $2 \mathrm{~cm}$ diameter view spot. They are evenly spaced from the inner wall to the outer baffle. The core plasma carbon content is measured using charge exchange recombination spectroscopy of fully stripped $\mathrm{C}^{+6}$ in both the visible [2,6] and the VUV [7]. The visible system has 40 chords densely spaced across the outer minor radius of the plasma. The VUV CER emission is measured using the midplane SPRED spectrometer, which crosses a beamline at a single radial location at a major radius $\mathrm{R}=1.8 \mathrm{~m}$. The midplane SPRED is also used to measure the brightness of lines from low charge states of carbon which arise from the outer scrape off layer (SOL) near the midplane. An IR camera viewing the lower divertor from above is used to measure the surface temperature and heat flux profile on the strike plate [8]. 
6 


\section{HELIUM AND DEUTERIUM L-MODE PLASMA COMPARISONS}

Similar L-mode lower single-null diverted (LSND) discharges with excess gas puffing have been run with both $\mathrm{D}$ and $\mathrm{He}$ gas fueling. A few basic discharge parameters as a function of time are shown in Fig. 1. The beam power in these two discharges is similar, however in discharge 94014 (deuterium), the beams are deuterium, while in 92333 the beam power is about $75 \%$ helium and $25 \%$ deuterium (needed for the CER diagnostics). The gas puffing programs shown are used to reduce the peak heat flux to the floor and reduce the divertor electron temperature. Note that prior to the programmed gas puff, the valve control voltage is responding to feedback control using $\mathrm{CO}_{2}$ laser interferometry and a programmed density rampup. The programmed puffs are different due to the fact that helium is fully recycling, while deuterium has a recycling coefficient somewhat less than one. The peak heat flux measured by the IR camera in the vicinity of the outer strike point is seen to drop to a low value for both discharges and in both cases the plate temperature never exceeds $360^{\circ} \mathrm{C}$. The electron temperature near the $\mathrm{X}$-point, measured by Thomson scattering at a vertical distance of $0.18 \mathrm{~m}$ above the strike plate, decreases to well below $5 \mathrm{eV}$ following gas puffing [3].

In order to assure that the helium discharges were dominantly helium, the experimental day followed a 16 hour bake of the vacuum vessel at $350^{\circ} \mathrm{C}$, and an entire day of operating with helium gas fueling. Spectroscopy data confirms that helium is the dominant species. Visible CER data from discharge \# 92342 (very similar to 92333) indicates that both edge and core helium densities are $\approx 50 \%$ of the local electron density, indicating a dominantly helium discharge. The $D_{\alpha}$ emission for all the divertor and inner wall channels is down by a factor of about 100 in the helium discharge, compared to the deuterium discharge. The divertor HeII recycling light is measured to be a factor of five stronger than the $D_{\alpha}$ emission. The divertor SPRED data in Table 1 shows that the HeII resonance line at $30.3 \mathrm{~nm}$ is a factor of 35 stronger in the helium discharge, compared to the $\mathrm{D}$ discharge. Line average densities are nealy equal in these discharges, at about $6 \times 10^{19} \mathrm{~m}^{-3}$ at $2000 \mathrm{~ms}$.

The divertor carbon is reduced to immeasurably low values in the helium plasma during the cold divertor phase. Figure 2 shows the core plasma carbon density and fraction $\left(\mathrm{n}_{C} / \mathrm{n}_{\mathrm{e}}\right)$ in these plasmas at a major radius of $1.8 \mathrm{~m}$ as a function of time measured using the CER emission in the VUV, along with the divertor CII $(658 \mathrm{~nm})$ brightness measured with the visible MDS spectrometer (sum of the seven lower divertor channels). During the L-mode phase prior to excess gas puffing, the helium plasma has a significantly lower carbon fraction than the deuterium plasma. Although the beam power in 92333 is lower than 94104 prior to gas puffing, 


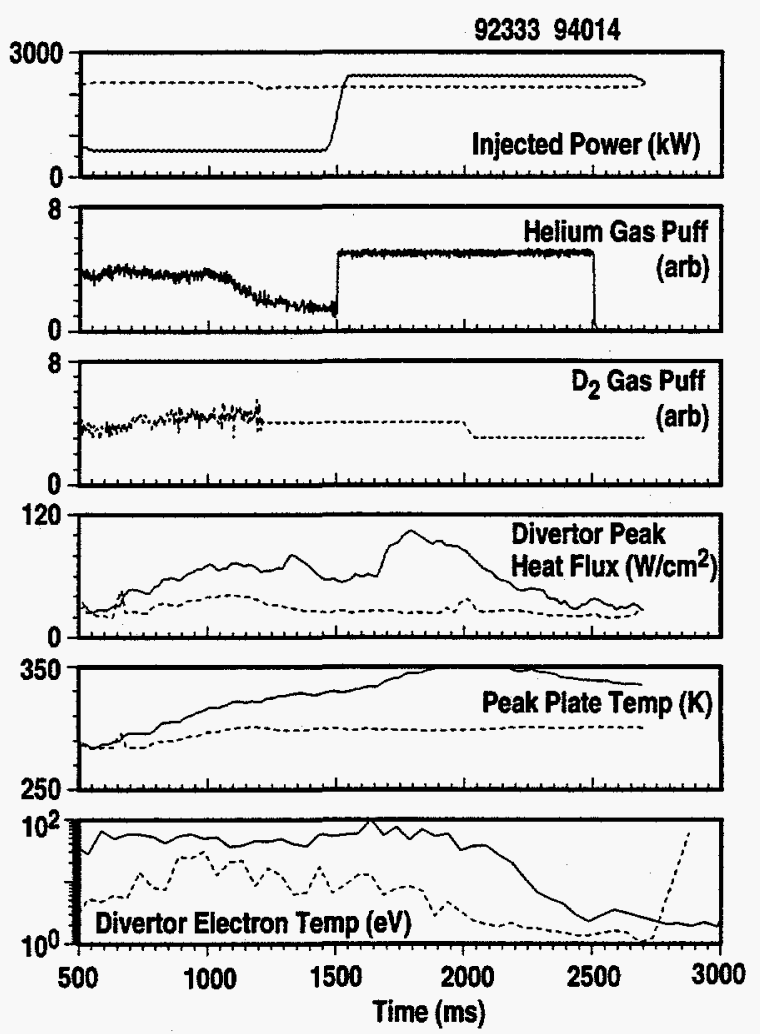

Fig. I. Time traces of several important discharge parameters for L-mode discharges with excess gas puffing with both $D_{2}$ (94014 - dashed lines) and He (92333 - solid lines) fueling are shown. The injected power consisted of about all deuterium beams for 94014 and 25\% deuterium and 75\% helium for 92333. The control voltages for the gas puffs in both discharges are shown, along with the peak divertor heat flux and plate temperatures measured with an IR camera. The electron temperature measured with Thomson scattering in the divertor, $17.5 \mathrm{~cm}$ above the divertor floor, illustrates that the divertor becomes very cold $(<5 \mathrm{eV})$ after the programmed gas puff is initiated in both discharges.

Table 1: Divertor carbon and helium VUV brightness measured with the divertor SPRED during cold divertor operation in helium and deuterium plasmas

\begin{tabular}{lcccc}
\hline & $\begin{array}{c}\text { CII } 133.5 \mathrm{~nm} \\
\left(\mathrm{ph} / \mathrm{s}-\mathrm{cm}^{2}-\mathrm{sr}\right)\end{array}$ & CIII 97.7 nm & CIV 155nm & HeII 30.3 nm \\
\hline D Plasma & $2.2 \times 10^{18}$ & $1.9 \times 10^{18}$ & $6.1 \times 10^{18}$ & $6.7 \times 10^{15}$ \\
He Plasma & $1.2 \times 10^{16}$ & $1.4 \times 10^{16}$ & $8.5 \times 10^{16}$ & $2.4 \times 10^{17}$ \\
\hline
\end{tabular}

comparison with other discharges at equal beam power also shows that the helium discharges have lower carbon content (Fig. 4). However, the fact that the peak heat flux to the floor is low throughout the deuterium discharge may indicate that the divertor is cold during the whole time shown [3]. After gas puffing has produced a cold divertor plasma ( $\mathrm{t}>2000 \mathrm{~ms}$ ), sufficiently cold that the ion impact energy on the divertor plate is well below the physical sputtering 
threshold [9], the carbon in the He plasma virtually disappears, while the carbon density in the D plasma rises slightly. The divertor carbon brightness remains approximately constant during the $\mathrm{D}$ discharge, while in the helium discharge the carbon emission drops dramatically after the programmed gas puff. Table 1 shows a comparison of divertor VUV carbon brightness observed using the divertor SPRED during the cold divertor phase of a helium and deuterium discharge.

A tangentially viewing TV camera with a CIII $(465 \mathrm{~nm})$ interference filter also shows a similarly strong reduction in the carbon content of the helium plasma compared to the deuterium plasma during the cold divertor phase. Edge brightness of low charge states of carbon observed with the midplane SPRED is shown in Fig. 2. Again, the helium plasma exhibits dramatically lower carbon emission compared to the deuterium plasma, however after the gas puff the helium plasma shows a small increase in midplane brightness, while there is a small decrease in the D plasma.

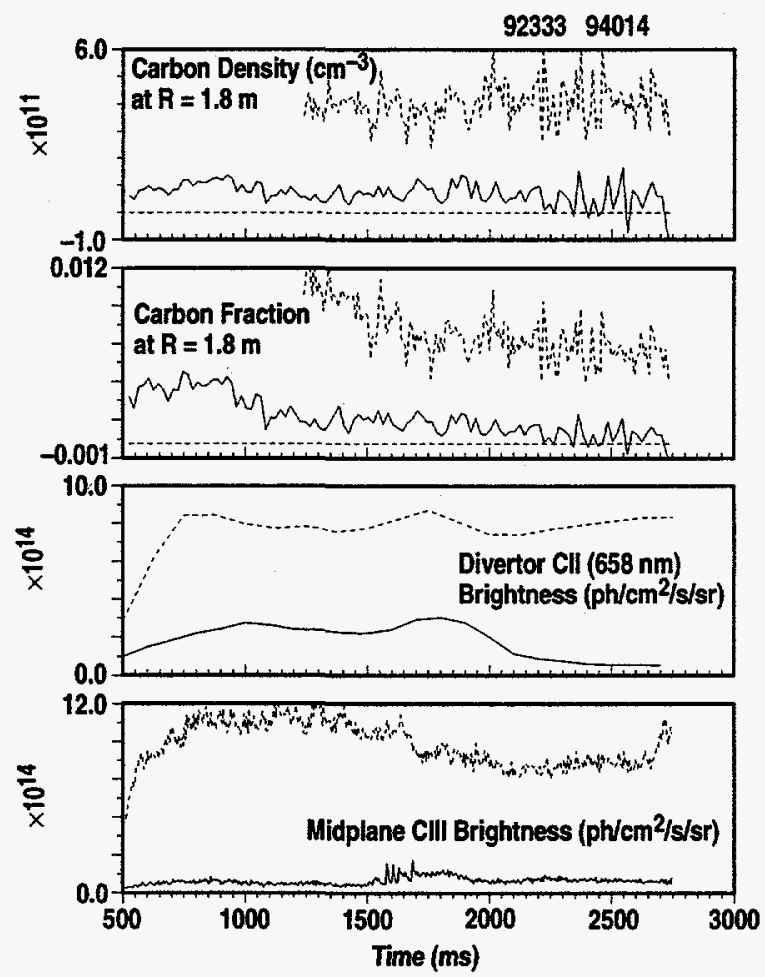

Fig. 2. Time traces from spectroscopic diagnostics for the two L-mode discharges are shown. The core carbon density at $\rho=0.1$ is calculated from the VUV CER emission measured with the midplane SPRED. The CIII VUV brightness is also measured with the midplane SPRED. The divertor CII brightness at 658 nm is measured with the MDS spectrometer. 
10 


\section{ELM FREE H-MODE PLASMAS}

Studies of high performance plasmas represent a major part of the DIII-D program. Because they are typically lower density with higher edge temperatures. they provide a contrast in edge conditions compared to the $\mathrm{L}$-mode discharges. Figure 3 shows a few important parameters as a function of time for two discharges, one helium and one deuterium, each with an ELM free $\mathrm{H}-$ mode period beginning at $1900 \mathrm{~ms}$ and lasting through $2200 \mathrm{~ms}$. These discharges are double null divertor (DND), high triangularity discharges and both reach VH-mode energy confinement levels [10] by $2200 \mathrm{~ms}$ ( $\tau_{\mathrm{E}} \approx 300 \mathrm{~ms}$ ). Prior to the $\mathrm{H}$-mode transition at $1900 \mathrm{~ms}$, the L-mode phases are similar, except that 92280 is fueled by a deuterium gas puff and heated by deuterium beams while 92283 is fueled by a heluim gas puff but heated by the same deuterium beams. During this period they both have low line averaged denisty $\left(\approx 2 \times 10^{19} \mathrm{~m}^{-3}\right.$ ). To precondition the walls and produce a high helium content plasma, the two discharges preceeding 92283 were helium fueled discharges, ohmically heated. Four Li pellets were injected into each of these preconditioning discharges, to reduce the deuterium recycling from the wall $[11,12]$. Visible CER data for helium is not available to verify the local helium density, however, other supporting evidence that 92283 is predominantly helium is provided in Fig. 3. During the L-mode phase, the inner wall $\mathrm{D}_{\alpha}$ brightness is an order of magnitude lower in the He discharge. The ratio of HeII $25.6 \mathrm{~nm}$ charge exchange emission measured by the midplane SPRED, normalized to the calculated beam attenuation factor, for the helium (92283) to deuterium (92280) discharges is maintained at a factor of 6 throughout the discharge. In the He discharge, the recycling intensity of HeII ( $468 \mathrm{~nm}$ ) compared to $\mathrm{D}_{\alpha}$ on the inner wall is observed to be about 5 during the L-mode phase, similar to the L-mode case discussed above where CER helium densities were available. The neutron production is down by a factor of four in the helium discharge, while the stored energies are nearly identical during the VH phases of these two discharges, and the heating beam input is identical. The reduced neutron production is indicative of a dominantly helium ion core plasma.

Carbon content of the core plasma is seen to rise quickly during the ELM free phase in both discharges. The carbon content in the edge of core plasma (normalized radius $\rho=0.72$ ), and the divertor CII and midplane CIII brightness are shown as a function of time for these two discharges in Fig. 4. The midplane SPRED shows that core carbon $(\rho=0.11)$ also rises during this phase. As seen in the previous L-mode case discussed above, during the L-mode phase of these discharges the helium discharge has a much lower carbon content. However, after the $\mathrm{L}-\mathrm{H}$ transition the core content of both discharges rises quickly, but the rate of increase is seen to be 
somewhat faster in the helium discharge. The divertor $\mathrm{CII}$ and midplane CIII brightnesses are not appreciably different between these two discharges.
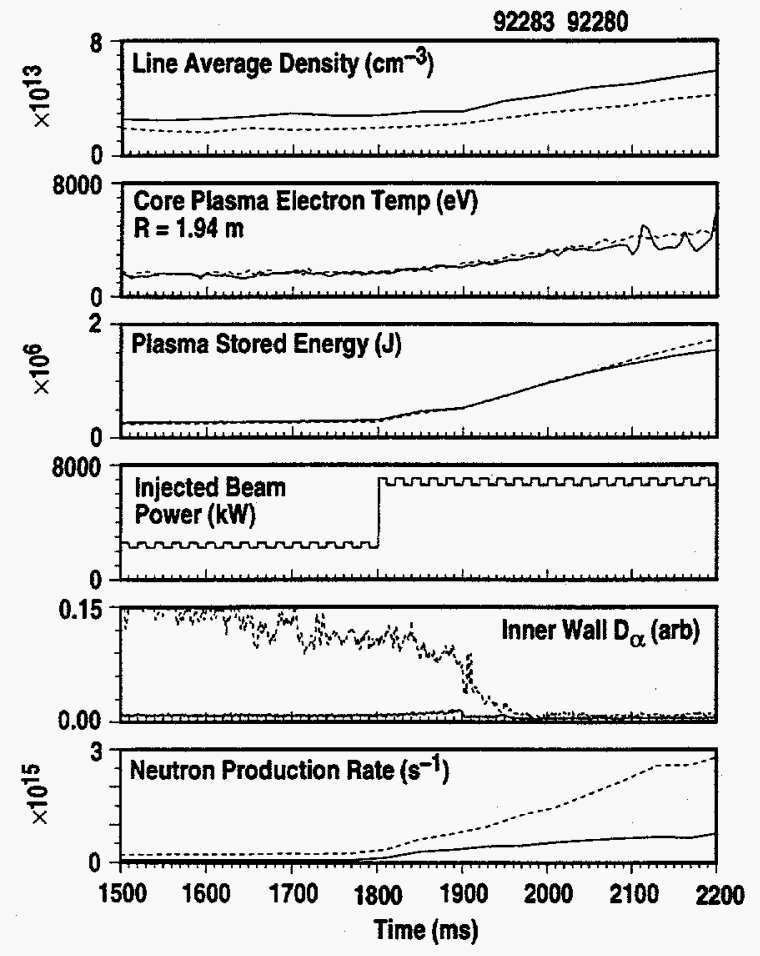

Fig. 3. Discharge parameters for the helium ( 92283 - solid lines) and deuterium (92280 - dashed lines) discharges with long ELM free periods are shown as a function of time. The line averaged density measured with interferometry, core plasma electron temperature measure with Thomson scattering, the stored energy from EFIT analysis of magnetic data, the injected power, the $D_{\alpha}$ recycling light measured at the inner wall, and the measured neutron production rates are shown. The ELM free $H$-mode period begins at $1900 \mathrm{~ms}$ and lasts through $2200 \mathrm{~ms}$.

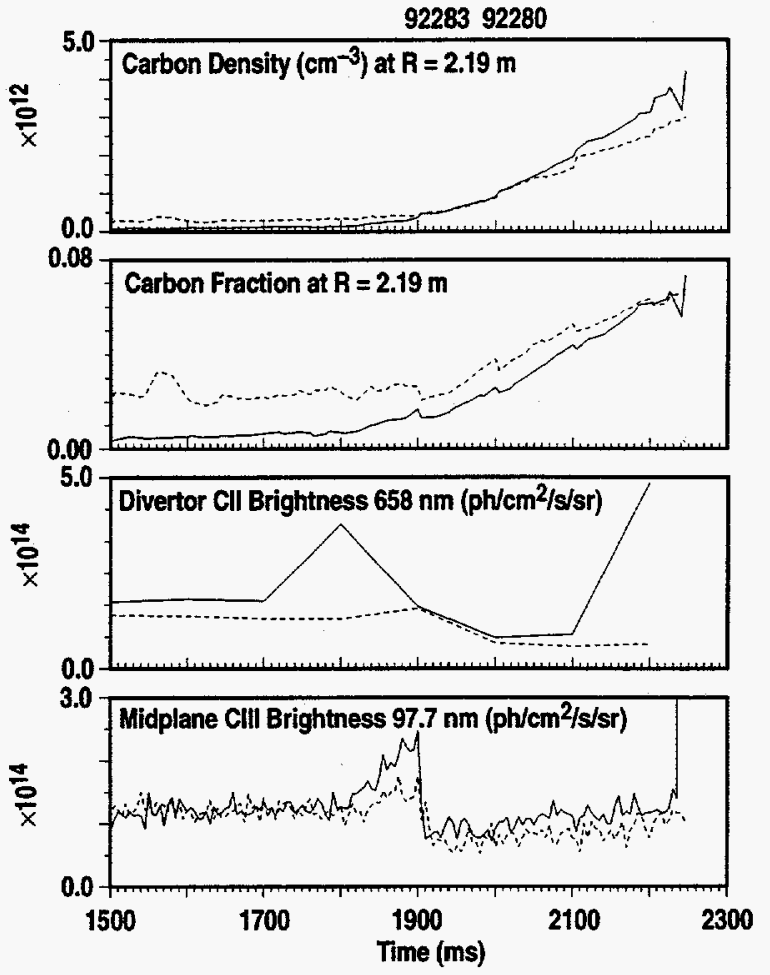

Fig. 4. Spectroscopic data from the helium (92283solid lines) and deuterium (92280 - dashed lines) discharges with long ELM free periods are shown as a function of time. The carbon densities and fractions measured using visible CER at $R=2.19 \mathrm{~m}(\rho=0.72)$ , the divertor CII emissivites at $658 \mathrm{~nm}$ and the midplane CIII emissivites at $97.7 \mathrm{~nm}$ are shown 


\section{DISCUSSION ON THE IMPLICATION FOR CARBON SOURCES: L-MODE}

In L-mode discharges, the core carbon content appears to be unrelated to the brightness of $\mathrm{CIII}$ at the outer midplane. From the time traces shown in Figs. 2 and 4 the midplane brightness of CIII in the DND discharges is essentially independent of main ion species, and is about equal to the emssivity of CIII in the LSND helium discharge. The LSND deuterium discharge has a brightness about an order of magnitude higher than the other three cases. Both DND discharges are at a low line average density during the L-mode phase, below $2 \times 10^{19} \mathrm{~m}^{-3}$, while the LSND discharges are above $6 \times 10^{19} \mathrm{~m}^{-3}$. The core carbon fraction, though, is clearly related to the main ion species. The outer midplane CIII brightness is indicative of the flux of carbon into the plasma at the outer midplane due to charge exchange neutral sputtering from the outer wall. Apparantly core carbon content is not related to the charge exchange sputtering from the outer main chamber wall in these L-mode plasmas. However, strong poloidal and toroidal asymmetries may exist in the carbon influx from the main chamber wall, which are not accounted for in the above argument.

Because the divertor carbon brightness shown in Fig. 2 for the gas puffed helium discharge is well correlated with the core plasma carbon content, it is suggestive that divertor sources are determining the core plasma carbon content. In the deuterium discharge, the fact that the divertor carbon brightness and core carbon content are unchanged after the transition to the cold divertor, even though the divertor plasma temperatures are reduced to levels well below the threshold for physical sputtering, would indicated that chemical erosion is playing an important role. The lower content in the helium discharge would support this conclusion. The reduction in divertor brightnesses and in core plasma content following the transition to a cold divertor in the helium discharge would suggest physical sputtering by either ions or charge exchange neutrals is playing a role prior to the transition, since chemical sputtering should be low throughout the helium discharge. MDS spectra of CII indicates that the CII brightness from the channel viewing the outer strike point accounts for about $70 \%$ of the sum of all the divertor channels, and also shows the most dramatic decrease after the gas puff induces cold divertor operation. This result indicates that sputtering at the outer strike point, most likely due to ion impact, is an important source during attached helium L-mode. However, CII spectra from the deuterium L-mode show a fairly uniform distribution of brightnesses across the divertor before gas puffing, becoming even more uniform after gas puffing has induced a cold divertor. Such uniformity is indicative of a more uniform source from the plate, as would likely be true for sources resulting primarily from incident neutrals, such as either charge exchange sputtering or chemical sputtering. 
Spectroscopic data from ELMing $\mathrm{H}$-mode discussed in Ref. [13] implies that physical sputtering by charge exchange neutrals dominates the divertor source during deuterium operation. In ELMing $\mathrm{H}$-mode deuterium discharges, with both attached and detached divertors, the molecular CD is usually below the detection limit using the MDS spectrometer. However the CI line at $833 \mathrm{~nm}$ is readily observable, and indicates that the carbon flux to the plasma is dominated by neutral carbon, rather than by molecular carbon. Detailed analysis indicates that in these ELMing H-mode deuterium discharges the flux of carbon atoms to the plasma is in the neighborhood of $5 \times 10^{20} \mathrm{~m}^{-2} \mathrm{~s}^{-1}$ while the flux of molecular carbon is below $2 \times 10^{19} \mathrm{~m}^{-2} \mathrm{~s}^{-1}$. This implies that physical sputtering by charge exchange neutrals dominates the divertor source during deuterium operation, which is counter to the discussion in the previous paragraph for helium discharges. Detailed measurements of charge exchange neutral flux and energy spectrum at the divertor walls have not been carried out on DIII-D. 


\section{DISCUSSION ON THE IMPLICATION FOR CARBON SOURCES: ELM FREE H-MODE}

While the data for the L-mode phases of these discharges show significant differences in the behavior of helium and deuterium discharges, the ELM free VH-mode phases of helium and deuterium discharges show remarkable similarities. The rate of increase of core carbon is dramatic during VH-mode in both cases, and divertor CII signals are comparable, both in total intensity and in the distribution, with the peak CII signal occuring at the outer strike point. Tangentially viewing TV data of the divertor CIII brightness in these two discharges (not shown) are also remarkably similar. In these discharges, the SOL is low density, and the divertor is in a low recycling regime. Typically this produces fairly high electron temperatures, $T_{e} \approx 50 \mathrm{eV}$ at the plate [12], resulting in efficient physical sputtering by ions impacting along the open field lines. In Ref. [13] it is shown that in a ELM free discharge, the measured erosion rate of carbon at the outer strike point is about equal to the rate of rise of carbon content in the core plasma. It has also been shown that the rate of rise of carbon in the core accounts for most of the rate of rise of the electron density [2]

One dimensional modeling of the divertor and SOL using the NEWT1D code [14] under low recycling conditions indicates qualitatively that such low density, high temperature divertor plasmas can produce significant backflow of impurity ions from the divertor to the midplane. Two cases have been modeled, the only difference is a change in the particle and power flux from the core plasma into the flux tube, as would be expected at the sudden change in edge transport that occurs at an L-H transition. The particle flux was reduced from $1.9 \times 10^{21} \mathrm{~s}^{-1}$ to $1.1 \times 10^{21}$ $\mathrm{s}^{-1}$ and the power flow from 3.3 MW to 2.5 MW. From these code calculations it is seen that the sum of the two primary forces on divertor carbon impurity ions, the frictional force from the primary ion flow toward the divertor and the $\nabla \mathrm{T}_{\mathbf{i}}$ force from the primary ion temperature gradient pushing carbon ions toward the midplane, goes from being strongly toward the plate over most of the outer divertor leg, to away from the plate over the entire divertor leg. The result is a dramatic increase in the total carbon that is transported from the strike plate to the midplane. In the high flux case, essentially no carbon reaches the midplane, but in the case with lower flux, the midplane carbon density reaches about $1 \%$ of the total ion density, reasonably close to the values of a few percent observed in the SOL of ELM-free discharges. 


\section{CONCLUSIONS}

Taken together, the L-mode data presented imply that the divertor region is the most important source of carbon for core contamination during L-mode operation, and that both charge exchange sputtering and chemical sputtering may play some role. Analysis of spectroscopic data presented in Ref. [13] indicates that in ELMing $\mathrm{H}$-mode deuterium plasmas physical sputtering, probably from charge exchange neutral flux to the divertor wall, is more important. More data is needed to determine which of these mechanisms is predominant in these L-mode discharges, since the dramatic reduction of carbon content in the helium plasmas, especially in the cold divertor phase, would tend to suggest that chemical sputtering is important for deuterium discharges.

In ELM free discharges, the data suggests that physical sputtering by ions flowing to the strike plate is the key source of carbon to the core plasma. The backflow of carbon to the core, combined with the improved confinement in the ELM free phases, results in a strong build up of carbon in the core, and has been shown in previous work to be responsible for a large fraction of the total electron density increase during these phases [12,2].

Obviously, if divertor design changes are to be implemented to reduce net recycling and core contamination, the divertor wall plasma facing material must be chosen with care. In highperformance, high-confinement phases, high divertor temperatures and low divertor densities result in high plate sputtering and impurity backflow. Thus, to keep core radiation under control, low $\mathrm{Z}$ materials are necessary. Because thermal loads, even in today devices, are high, graphite is clearly the low $\mathrm{Z}$ material of choice. However radiative divertor techniques devised to reduce divertor thermal loads, and to reduce physical sputtering, do not seem to reduce the carbon content in DIII-D. High Z refractory metals, such as tungsten, are resistant to chemical sputtering and have a high energy threshold for physical sputtering. Tungsten appears to be material of choice under these conditions. A clear solution to the divertor plasma facing material problem requires that radiative divertor techniques for high performance plasmas must be developed. 


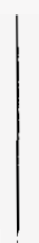




\section{REFERENCES}

[1] R.D. Wood, R.C. Isler, S.L. Allen, M.E. Fenstermacher, C.J. Lasnier, A.W. Leonard, W.P. West, Proc. of the 23rd European Physical Society Conference on Controlled Fusion and Plasma Physics, Kiev, Volume 20C (1996) 763.

[2] M.R. Wade, et. al., Proc. of the 23rd European Physical Society Conference on Controlled Fusion and Plasma Physics, Kiev, Volume 20C (1996) 283.

[3] T.W. Petrie et. al., Nucl. Fusion 37 (1997) 321.

[4] D.G. Whyte, et al., J. Nucl. Mater. 241-243 (1997) 660.

[5] C.P.C. Wong, et al., "Divertor Materials Evaluation System (DiMES)," ICFRM8, Sendai, Japan, to be published in J. Nucl. Mater.

[6] P. Gohil, et al., Proc. 14th Symposium on Fusion Energy, San Diego, 1992 (IEEE, New York, 1992), Vol. 2, 1199.

[7] D.G. Whyte, et al., Nucl. Fusion 38 (1998) 387.

[8] D.N. Hill, et al., Rev. Sci. Instrum. 59 (1988) 1878.

[9] W. Eckstein, C. Gargia-Rosales, J. Roth, et al., "Sputtering Data," IPP, Garching, Germany, IPP 9/82, 1993.

[10] G.L. Jackson, J. Winter, T.S. Taylor, et al., Phys. Rev. Lett. 67 (1991) 3098.

[11] G.L. Jackson, et al., J. Nucl. Mater. 241-243 (1997) 655.

[12] D.K. Mansfield, et al., Phys. Plasmas 3 (1996) 1892.

[13] D.G. Whyte, et al., "Divertor Erosion in DIII-D," this conference.

[14] R.B. Campbell, et al., J. Nucl. Mater. 196-198 (1992) 426. 
20 


\section{ACKNOWLEDGMENT}

Work supported by U.S. Department of Energy under Contracts DE-AC03-89ER51114, DEAC05-96OR22464, W-7405-ENG-48, DE-AC02-76CH03073, and Grant DE-FG03-95ER54294. 